frequently-relapsing/steroid-refractory disease course, sustained remission is more likely if immunomodulators/biologics are introduced earlier. However, many patients experience a quiescent course even without immunomodulators, and should not be exposed to the risks of unnecessary immunosuppression. Previous attempts to personalize treatment from diagnosis have been limited by our inability to reliably predict disease behaviour. Using microarray-based whole genome expression profiling, we have recently identified a common gene expression signature in CD8 T cells in two different autoimmune diseases (ANCA-associated vasculitis and SLE) that correlates with disease course. We attempted to determine if a similar signature existed in CD or UC.

Methods We performed whole genome expression analysis of CD8 T cells isolated from the blood of 60 patients with active CD or UC before commencing treatment. Prospective follow-up was performed for up to 700 days. Patients were managed using a conventional 'step-up' approach by clinicians blinded to the microarray results.

Results Microarray analysis of CD8 T cell gene expression data revealed the presence of two distinct subgroups within each disease cohort. Comparison of the genes that were differentially expressed between the subgroups in CD and UC demonstrated highly significant overlap $\left(p<1 \times 10^{-300}\right)$. Patients belonging to the equivalent subgroup in both diseases (termed IBD1) experienced a more aggressive disease course than those in the other subgroup (termed IBD2). This was characterised by a greater requirement to introduce immunomodulators or perform surgery ( $p=0.0025$ (CD), $p=0.0006$ (UC)) and the necessity for more such escalations over the follow-up period $(p=0.022$ (CD), $p=0.006$ (UC)). Patients could not be assigned to these subgroups using other contemporaneous clinical or laboratory parameters. In both diseases, this stratification was superior to previously described methods of predicting outcomes.

Conclusion Gene-expression profiling of CD8 T-cells isolated from blood can stratify patients with both UC and CD at diagnosis according to subsequent disease behaviour and represents a significant step towards personalised therapy.

Competing interests None.

Keywords gene expression profiling, microarray, prognostic biomarkers.

\title{
OC-122 A CD8 T CELL GENE EXPRESSION SIGNATURE PREDICTS DISEASE BEHAVIOUR IN INFLAMIMATORY BOWEL DISEASE
}

\author{
doi:10.1136/gut.2011.239301.122
}

J C Lee, ${ }^{1,2^{*}} \mathrm{P}$ Lyons, ${ }^{1}$ M Parkes, ${ }^{2}$ K G Smith ${ }^{1}$ Cambridge Institute for Medical Research, University of Cambridge, Cambridge, UK; ${ }^{2}$ Department of Gastroenterology, Addenbrooke's Hospital, Cambridge, UK

Introduction The natural history of Crohn's disease (CD) and ulcerative colitis (UC) is variable. In patients with a 